\title{
Cross-Sectional Scanning Tunneling Microscopy Studies of Lattice-Matched InGaAs/InP Quantum Wells: Variations in Growth Switching Sequence
}

\author{
H. A. McKay and R. M. Feenstra \\ Department of Physics, Carnegie Mellon University, Pittsburgh, Pennsylvania 15213 \\ P. J. Poole and G. C. Aers \\ Institute for Microstructural Sciences, National Research Council of Canada, Ottawa, K1A0R6, Canada
}

\begin{abstract}
Cross-sectional scanning tunneling microscopy (XSTM) has been used to study variations in the source gas switching sequences in lattice-matched InGaAs/InP heterostructures grown by chemical beam epitaxy. Using finite element analysis, changes in well-barrier interface strain can be understood in terms of As/P exchange and As memory effect in the growth chamber. Results from annealed samples indicate a greater interdiffusion of group-V species than group-III species.
\end{abstract}

\section{INTRODUCTION}

$\mathrm{In}_{0.53} \mathrm{Ga}_{0.47} \mathrm{As} / \mathrm{InP}$ quantum well (QW) structures have applications in optoelectronic devices such as transistors [1] and photodetectors [2]. Though this material system has been used successfully in devices for several years there are still concerns regarding the material composition at the InP-on-InGaAs and InGaAs-on-InP interfaces. Typically, both the group III and group V species compositions will change when going across a QW-barrier interface, allowing the possibility of significant strain developement if any mixing occurs. This can result in a modification of optical emission energies, and the developement of roughness that can lead to relaxation and broadening of photoluminescence (PL) spectra. These deviations from ideality may adversely affect the optical and electronic properties of the heterostructures [3].

In chemical beam epitaxy (CBE) of InGaAs heterostructures, interface composition may be adjusted by changing parameters in the group III and group V source gas switching sequence. For instance, to achieve acceptably abrupt QW interfaces, it is necessary to include a growth interrupt when changing from well to barrier growth and vice versa.

Earlier studies of these effects used indirect measurements of the interfaces, such as PL [9], and high resolution X-ray diffraction [4] to investigate and optimize the switching sequences used to grow InGaAs/InP heterostructures. Cross-sectional scanning tunneling microscopy (XSTM) has not often been used for such studies [5]. In this paper we are able to use XSTM to observe the effect of the gas switching sequence on the interface structure of a set of InGaAs/InP QWs grown in a single run, so all of the changes observed can be attributed to the switching sequences used. This allows us to make direct measurements of the strain in the layers on a microscopic scale and to separate effects at each interface.

In this work $\mathrm{In}_{0.53} \mathrm{Ga}_{0.47}$ As QWs were grown using four different gas switching sequences. Following previous XSTM work, we interpret contrast variations in topographic images in terms of strain relaxation of the cleaved surfaces. In each of the switching sequences, As/P exchange appears to be the dominant mechanism at the interfaces. As-to-P exchange causes the InGaAs-on-InP interface to be compressively strained and P-to-As exchange causes the InP-on-InGaAs interface to be under tensile strain. The amounts of tensile and compressive strain vary depending on the parameters used for each gas switching sequence.

\section{EXPERIMENT}

The wafers studied here were grown via chemical beam epitaxy on [001]-oriented S-doped InP substrates in a RIBER CBE $32 \mathrm{P}$ reactor at $510^{\circ} \mathrm{C}$. The group III sources, TEGa and TMI were introduced into the chamber without carrier gas through a fast switching low temperature injector. The group V sources were pure $\mathrm{AsH}_{3}$ and $\mathrm{PH}_{3}$ and were cracked at $850^{\circ} \mathrm{C}$ in a fast switching high temperature injector to produce predominantly $\mathrm{As}_{2}$ and $\mathrm{P}_{2}$. Both cells gave switching transients significantly less than 1 second. The grown layers were doped n-type with silicon to a concentration of $2 \times 10^{18} \mathrm{~cm}^{-3}$ using $\mathrm{SiBr}_{4}$.

The heterostructure consisted of a $100 \mathrm{~nm}$ thick layer of lattice matched InGaAs grown on an InP buffer, followed by a stack of ten $8 \mathrm{~nm}$ thick $\mathrm{In}_{0.53} \mathrm{Ga}_{0.47} \mathrm{As} \mathrm{QWs}$, separated by $16 \mathrm{~nm} \mathrm{InP}$ barriers and capped with $\mathrm{InP}$. The $\mathrm{InP}$ was grown at a rate of $0.64 \mu \mathrm{m} /$ hour, and the InGaAs at $1 \mu \mathrm{m} /$ hour. Four different gas switching sequences (A, B, C, D) were used to grow sets of quantum wells, as listed in Table I.

Sequence A is our conventional gas switching procedure. Barrier (or well) growth is terminated with a 10 s purge of the group III elements to smooth the surface followed by a $5 \mathrm{~s}$ purge of the group $\mathrm{V}$ element to completely remove any residual group $\mathrm{V}$ species from the 
growth chamber. The group V species for the next layer was then introduced, followed $1 \mathrm{~s}$ later by the group III elements to start the growth of the next layer. The sequences used on both sides of the QWs were symmetrical.

Sequence B was used by Rongen et al. [6] where they predict an optimum and symmetric interface. In this sequence the InP surface is allowed to smooth for $2 \mathrm{~s}$, the group V species are then simultaneously swapped and $1 \mathrm{~s}$ later the TMI and TEGa introduced. At the other interface the InGaAs was smoothed for $2 \mathrm{~s}$ before the group $\mathrm{V}$ species are simultaneously swapped and the TMI introduced. This was predicted [6] to result in 1 monolayer (ML) of InAs $\mathrm{s}_{0.5} \mathrm{P}_{0.5}$ at both $\mathrm{InP} / \mathrm{InGaAs}$ interfaces of the QW.

Sequence $C$ is taken from Ref. [7] where it was optimized for the growth of high quality multi-QWs. The switching sequence when going from InP to InGaAs is the same as in sequence $B$, but with a 5 s smoothing time, so the result at the InGaAs-on InP interface should be very similar. The other interface is somewhat different, with a $10 \mathrm{~s}$ smoothing time followed by the exposure of the InGaAs surface to $\mathrm{PH}_{3}$ for $10 \mathrm{~s}$ before introducing the TMI to grow the InP barrier. The purpose of this $\mathrm{PH}_{3}$ soak was to avoid $\mathrm{As}$ incorporation in the barrier material.

The final sequence, $D$, is very similar to sequence $A$ but with the group III species introduced $1 \mathrm{~s}$ before, not after, the introduction of the new group $\mathrm{V}$ species. This was shown to reduce the effects of As/P exchange at the QW interfaces $[9,10]$ when grown by gas source MBE. Details of the switching sequences are summarized in Fig. 1.

In addition to varying the switching sequence, portions of the wafers were annealed $\left(800^{\circ} \mathrm{C}, 120 \mathrm{sec}, \mathrm{N}_{2}\right.$ ambient, InP proximity cap) in a conventional tube furnace. Anneal parameters were chosen to promote significant interdiffusion between the barriers and the quantum wells.

Cross-sectional STM measurements were performed in an ultrahigh vacuum chamber with a base pressure $<5 \times 10^{-11}$ torr. Tungsten probe tips were electrochemically etched and cleaned via electron bombardment. Samples were cleaved in situ to expose (110) surfaces. STM images were acquired with a constant tunnel current of $0.1 \mathrm{nA}$ at sample biases in the range of 1.5 to 2.5 volts. Several different probe tips were used for as-grown and annealed samples.

\section{RESULTS AND DISCUSSION}

Figure 1 shows an STM image of part of the as-grown QW stack. This is an empty-state image so only atoms on the group III sublattice are imaged. The wells appear as mottled stripes while InP barriers appear as broader homogeneous bands. The mottling in the wells is due to compositional fluctuations in the InGaAs alloy. The letter denoting the particular growth switching sequence used for each well is displayed beneath the correspond- ing alloy layer. The growth direction in every image displayed in this paper is from right to left. There are several defects scattered over the image (isolated dark or bright 1-2 $\mathrm{nm}$ size features). Most of these are likely due to residual gas contamination from the UHV system, however, a few are probably Si dopant atoms. The plot below the image shows the line scan average of the image. Peaks are seen at the InGaAs-on-InP interfaces and troughs at the InP-on-InGaAs interfaces. An atomic step occurs at the extreme left edge of the surface area. Examination of the average line scan shows that the entire QW stack appear to bulge out of the surface by a few tenths of an angstrom.

It was shown in Refs. [16-18] that in empty-state images of $\operatorname{In}_{\mathrm{x}} \mathrm{Ga}_{1-\mathrm{x}} \mathrm{As}_{\mathrm{y}} \mathrm{P}_{1-\mathrm{y}}$ surfaces, tip height changes due to strain relaxation effects contribute more to the STM image than do variations in sample electronic structure (the latter have magnitude typically $\lesssim 0.1 \AA$ ). Therefore, changes in tip height at barrier-well interfaces can be attributed mainly to strain effects.

Finite element analysis (FEA) can therefore be used to deduce information on the chemical composition of the heterostructure layers. In this method, one assumes some composition profile throughout the heterostructure, thus producing a strain profile in the heterostructure. One then introduces a free surface (i.e. the cleavage surface), and using FEA one computes the displacement of this surface as a function of position through the heterostructure. Comparison with the experimental results is then used to refine the assumed compositional profile.

The FEA computer code used here was developed specifically for this application, following standard algorithms [8], and includes the full anisotropic elastic properties of the cubic semiconductors. Comparison with commerically available packages (Algor) have been make to ensure the accuracy of the code.

From Fig. 1 it can be seen that each switching sequence has produced a compressively strained layer at the InGaAs-on-InP interface and a tensilely strained layer at the InP-on-InGaAs interface. The background bowing in Fig. 1 suggests that the entire heterostructure is under compressive strain, probably due a small As background in the InP barriers caused by As carry over from the QWs. X-ray diffraction measurements also indicate a small average compressive strain for the heterostructure.

To understand the effects of the switching sequences used at the QW interfaces, average line scans of the XSTM images were fitted using the finite element model. Line scans are shown in Fig. 2 together with the fitted interface profiles. Four line scans were chosen for each QW set to indicate the degree of variation observed in the images. The fitting parameters were generated assuming that the dominant mechanism for the generation of strain at the interfaces was As/P exchange, and are given in Table II. Other mechanisms are discussed later in the paper. The thickness used for the interface layers are somewhat arbitrary, and were chosen to be $5 \AA$ to fit the $2.5 \AA$ grid used for the finite element calculation. 
Figure 3 shows a summary plot of the average tip displacements over the interfaces. Positive displacements for measurements taken over the InGaAs/InP interfaces and negative displacements for measurements over InP/InGaAs interfaces. Uncertainty is calculated using the error on the mean.

Two characteristic features are always observed in the QWs investigated here, a peak in the surface height at the InGaAs-on-InP interface, and a dip at the InP-onInGaAs interface. It is the magnitude of these features that vary considerably between switching sequences. We will start by looking at the InGaAs-on-InP interface. In all cases the InP surface is first smoothed using a growth interruption in a phosphorus atmosphere. The length of this smoothing time is not expected to affect the STM images; it determines interface roughness on the monolayer scale. If this is true then sequences $B$ and D should be equivalent, whereas sequences $\mathrm{A}$ and $\mathrm{C}$ highlight phosphorus memory effects and $A s / P$ exchange effects respectively. Sequences A, B, and C can all be fitted using the same $0.5 \mathrm{~nm} \mathrm{InAs_{0.65 }} \mathrm{P}_{0.35}$ interface layer, which means that the $5 \mathrm{~s}$ purge of the phosphorus for sequence $\mathrm{A}$ has no apparent effect in increasing the degree of As/P exchange or phosphorus carry over into the InGaAs QW. This result means that there are no memory effects for phosphorus in our growth system, or that the time scale to observe these effects is considerably longer than the 5 s purge time used here. Rongen et al. [6] had suggested a single monolayer of $\operatorname{InAs_{0.5}} \mathrm{P}_{0.5}$ at this interface, about half of what is observed here. The amount of As/P exchange is dependent upon the growth conditions used, e.g. growth temperature and group V flux, and could explain the difference. We had observed less As/P exchange in an earlier sample [17] grown under different growth conditions using a slower switching group III gas injector. Sequence D requires a slight reduction in the amount of As/P exchange to obtain a good fit, as suggested by earlier work [9]. The presence of the group III species on the surface have reduced the As/P exchange process, but only to a small degree, and the As is still able to exchange with $\mathrm{P}$ through this layer.

Greater differences are observed in the behaviour of the InP-on-InGaAs interface when changing the switching sequences. Sequence A can be fitted by a tensile $0.5 \mathrm{~nm} \mathrm{In}_{0.532} \mathrm{Ga}_{0.468} \mathrm{As}_{0.6} \mathrm{P}_{0.4}$ layer due to the exposure of the InGaAs to phosphorus for $1 \mathrm{~s}$ before growing the InP. The tensile layer observed for sequence $\mathbf{B}$, $\mathrm{In}_{0.532} \mathrm{Ga}_{0.468} \mathrm{As}_{0.7} \mathrm{P}_{0.3}$, has less phosphorus than with sequence $\mathrm{A}$ since the phosphorus and indium are introduced simultaneously. The presence of this tensile layer in sequence B clearly disagrees with the assumptions made by Rongen et al. [6] where they assume that the As/P substitution can be ignored. Sequence $C$ was specifically developed to avoid As incorporation into the InP barrier material (discussed later in this paper) [7]. The long exposure of the InGaAs leads to considerable As/P exchange, and the formation of a highly strained $0.5 \mathrm{~nm} \mathrm{In}_{0.532} \mathrm{Ga}_{0.468} \mathrm{As}_{0.2} \mathrm{P}_{0.8}$ layer. Sequence $\mathrm{D}$ results in the smallest degree of As/P exchange due to the deposition of the indium layer before the phosphorus, as expected.

Carry over of arsenic into the InP barriers following the growth of InGaAs has been observed using a number of growth techniques $[7,11,12,16]$. This has been attributed to two effects, a growth chamber memory effect where residual As remains in the chamber after growing As containing layers, and segregation of As from the InGaAs layer. The excess As in the InP barrier results in a compressively strained layer that decays in As composition away from the QW. Switching sequences $\mathrm{A}$ and $\mathrm{C}$ show no strong evidence for As carry over, whilst sequences B and $\mathrm{D}$ fit better with a carry over effect. This can be understood from the switching sequences used if the excess As is assumed to be due to segregation. Sequences A and $\mathrm{C}$ both expose the InGaAs surface to a phosphorus flux before growing the InP barrier, which then removes any excess As from the surface. The interesting comparison is between sequences A and D where the As is switched out of the chamber for 6 and $5 \mathrm{~s}$ respectively before the group III species is introduced. In this case any memory effect of the growth chamber would be expected to be the same, so any resulting As tail would be the same, but these two samples are quite different. This result means that the As must be coming from the sample itself for sequence $\mathrm{D}$, and the way that the InGaAs surface is treated clearly modifies the As carry over. Consistent with the suggestion of Rigo et al. [7], the long phosphorus soak used here reduced the amount of As carry over.

In all of the above discussion the mechanism for the formation of compressive and tensile spikes at the interfaces has been assumed to be As/P exchange. However, it is also possible to invoke exchange of the group III species. The main mechanism for this exchange would be In segregation. Indium segregation would produce an InGaAs QW where the leading edge is In rich, resulting in a region of compressive strain when switching from InP to InGaAs. At the other interface In segregation is meaningless, but $\mathrm{Ga}$ segregation would lead to a tensile layer. Studies into In surface segregation during CBE growth $[13,14]$ indicate that a monolayer of In floats on top of an InGaAs or InGaP layer, and that the composition of the ternary does not reach equilibrium until a full monolayer of In is reached. Adding a monolayer of InP on GaAs achieved the suitable amount of floating In when growing InGaP on InP [13]. This result means that growing InGaAs on InP (as is the case for our sample) would result in a uniform InGaAs composition and no compressive spike at the InGaAs on InP interface, consistent with the conclusions of Landesman et al. [14]. They also did not find strong evidence for Ga segregation into the InP barrier material, although it is not possible to completely rule this out based on our measurements. It is clear that the As/P exchange process does occur at the InP-on-InGaAs interface from the measurements of the QWs grown with sequence C. Possible Ga segregation effects could be masked by the As/P exchange, but nei- 
ther In or Ga segregation effects are required to explain our results in this paper.

Sequence D is nominally the same as that used in a previous study [17] where we were using an older group III gas injector that showed considerably longer switching transients. The group III species were also introduced into the chamber using $\mathrm{H}_{2}$ as a carrier gas. It is interesting to note that the line scans from that earlier work differ in two noticeable respects from those of sequence $\mathrm{D}$ in this work. Firstly the compressive layer peak at the InGaAs-on-InP interface was substantially lower in that work $(\approx 0.15 \AA$ compared to $\approx 0.25 \AA)$. Secondly, we observed a small dip before this peak due to a tensile layer. In the earlier work it was suggested that this could be due to the formation of an InGaP or InGaAsP layer when the InP surface was exposed to the In and Ga flux for the 1 $\mathrm{s}$ before the introduction of As. It should be noted that adding a tensile layer of the type included in that work next to the more compressive layers of sequence $D$ leads to only small changes in the calculated surface profile, and a dip is not evident. It is thus not possible to state whether there is a tensile layer formed at the InGaAs on InP interface for sequence $\mathrm{D}$, but it should be noted that the temporal behaviour of the group III fluxes would be expected to be significantly different between the two growths.

We now turn to discuss results from the annealed sample. Figure 4 shows an empty-state image of the QW stack. The line-scan average tip height is displayed below the image. From the image and the average line scan, it is apparent that annealing has had a pronounced effect on strain at the interfaces and within the QWs themselves. The InP-on-InGaAs interfaces for switching sequences $\mathrm{A}, \mathrm{B}$, and $\mathrm{D}$ now show compressive strain instead of the tensile strain seen in the as-grown heterostructure. Also, the mid-points of these wells is now depressed. The apparent well widths have broadened by over $50 \%$ compared to the as-grown wells, though the increased width for the $\mathrm{C}$ wells is less than this. The observation of compressive strain at the interfaces and tensile strain in the wells is consistent with previous results from $P$ ion-implanted QW heterostructures [17] in which the implantation occurs above the QWs. Data from the current work and Ref. [17] are consistent with As diffusing out of the well while $\mathbf{P}$ diffuses into the well. We can conclude that atoms from the group V sublattice interdiffuse more than atoms from the group III sublattice since we would see the opposite (or little) strain effects if group III interdiffusion was as great or greater than group V interdiffusion. This interpretation is consistent with the XRD results from Ryu et al. [19].

The upper interface for the type $\mathrm{C}$ wells still appears to be under tensile strain, though the magnitude of the dip at this interface has decreased. It may be that the strained $\mathrm{In}_{0.532} \mathrm{Ga}_{0.468} \mathrm{As}_{0.7} \mathrm{P}_{0.3}$ layer that was present at this interface from the original growth has reduced As/P interdiffusion during the anneal.

\section{SUMMARY}

A lattice-matched InGaAs/InP heterostructure was grown using four different gas-switching sequences. The heterostructure was characterized using XSTM and the results interpreted in terms of strain relaxation of the cleaved surface. Irrespective of the particular switching sequence, we find the InGaAs-on-InP interfaces are compressively strained and the InP-on-InGaAs interfaces are under tension. The compressive strain at the InGaAson-InP interface is likely due to $\mathrm{As} / \mathrm{P}$ exchange during the growth interrupt. Varying the $\mathbf{P}$ soak times had little effect on the overall strain for sequences A, B, and C. The presence of group III elements on the surface before exposure to As is found to As/P exchange in the wells grown with sequence $\mathbf{D}$.

Increased variation in the amount of tensile strain at the InP-on-InGaAs interfaces can be understood in terms of changes in the amount of time allowed for As/P exchange and As memory effect in the chamber. during growth.

The affects of thermal annealing are consistent with earlier XSTM results from ion-implanted multiple QW structures and with XRD results on annealed heterostructures from other workers [19]. We note that As/P interdiffusion appears to have been inhibited at InP-onInGaAs interfaces grown with sequence $\mathrm{C}$.

\section{ACKNOWLEDGMENTS}

This work was supported by the U.S. National Science Foundation, grant DMR-9985898.

[1] Wen-Huei Chiou, Hsi-Jen Pan, Rong-Chau Liu, ChunYuan Chen, Chih-Kai Wang, Hung-Ming Chuang and Wen-Chau Liu, Semicond. Sci. Technol. 17, 87 (2002).

[2] D. K. Sengupta, S. L. Jackson, D. Ahmari, H. C. Kuo, J. I. Malin, S. Thomas, M. Feng, G. E. Stillman, Y. C. Chang, L. Li, and H. C. Liu, Appl. Phys. Lett. 69, 3209 (1996).

[3] Y. Gusakov, E. Finkman, G. Bahir, and D. Ritter, Appl. Phys. Lett. 79, 2508 (2002).

[4] R. Benzaquen, A. P. Roth, and R. Leonelli, J. Appl. Phys. 79, 2640 (1996).

[5] S. Thomas, H. C. Kuo, A. P. Curtis, W. Wu, J. R. Tucker, and G. E. Stillmam, Conference Proceedings 1997 International Conference on Indium Phosphide and Related Materials, (IEEE, 1997), pp.312-15.

[6] R. T. H. Rongen, A. J. C. van Rijswijk, M. R. Leys, C. M. van Es, H. Vonk, and J. H. Wolter, Semicond. Sci. Technol. 12, 974 (1997). 
[7] C. Rigo, A. Antolini, C. Cacciatore, C. Coriasso, L. Lazzarini, and G. Salviati, J. Cryst. Growth 136, 293 (1994).

[8] D. S. Burnett, Finite Element Analysis, From Concepts to Applications, (Addison-Wesley, Reading MA, 1987).

[9] G. J. Shiau, C. P. Chao, P. E. Burrows, and S. R. Forrest, J. Appl. Phys. 77, 201 (1995).

[10] T. Anan, S. Sugou, K. Nishi, and T. Ichihashi, Appl. Phys. Lett. 63, 1047 (1993).

[11] W. Seifert, X. Liu, and L. Samuelson, Appl. Phys. Lett. 62, 949 (1993).

[12] T. Mozume, H. Kashima, K. Hosomi, K. Ouchi, H. Sato, H. Masuda, T. Tanoue, and I. Ohbu, J. Vac. Sci. Technol., B 13, 276 (1995).

[13] M. Mesrine, J. Massies, C. Deparis, N. Grandjean, and E. Vanelle, Appl. Phys. Lett. 68, 3579 (1996).

[14] J. P. Landesman, J. C. Garcia, J. Massies, G. Jezequel, P. Maurel, J. P. Hirtz, and P. Alnot, J. Vac. Sci. Technol., B 10, 1761 (1992).

[15] R. M. Feenstra, D. A. Collins, D. Z.-Y. Ting, M. W. Wang, and T. C. McGill, J. Vac. Sci. Technol. B 12, 2592 (1994).

[16] H. Chen, R.M. Feenstra, P.G. Piva, R.D. Goldberg, I.V. Mitchell, G.C. Aers, P.J. Poole, and S. Charbonneau, Appl. Phys. Lett. 75, 79 (1999).

[17] H. Chen, H.A. McKay, R.M. Feenstra, G.C. Aers, P.J. Poole, R.L. Williams, S. Charbonneau, P.G. Piva, T.W. Simpson, and I.V. Mitchell, J. Appl. Phys. 89, 4815 (2001).

[18] R.M. Feenstra, Physica B 273-274, 796 (1999).

[19] Sang-Wan Ryu, Byung-Doo Choe, and Weon Guk Jeong, Appl. Phys. Lett. 71, 1670 (1997).

FIG. 1. Empty-state STM image of several of the as-grown quantum wells (top). The wells appear as mottled bands. Each well is labelled with the particular gas switching sequence used during growth. The grey-scale range is $1.0 \AA$ and the sample bias voltage was $+1.5 \mathrm{~V}$. Line scan average of the STM image (bottom)

FIG. 2. Representative average line scans from each switching sequence. The theory profiles were calculated from the finite element model.

FIG. 3. Average maximum tip displacements over the InGaAs-on-InP interfaces (positive values) and over the InP-on-InGaAs interfaces (negative values).

FIG. 4. Empty-state STM image of the annealed quantum wells (top). The Each well is labelled with the particular gas switching sequence used during growth. The grey-scale range is $0.8 \AA$ and the sample bias voltage was $+2.0 \mathrm{~V}$. Line scan average of the STM image (bottom). The strain at the InP-on-InGaAs interfaces for sequences A, B, and D has become compressive compared to the tensile strain in the as-grown quantum wells. The strain for sequence $\mathrm{C}$ has remained tensile.
Table I. Details of growth switching sequences A, B, C, and D. The direction of growth is from top to bottom.

\begin{tabular}{|c|c|c|}
\hline \multicolumn{3}{|c|}{ Segझance A } \\
\hline Batra俍 & 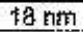 & \\
\hline & 105 & Vent IM! \\
\hline & 55 & Vert $\mathrm{PH}_{3}$ \\
\hline & 15 & foun $\mathrm{AsH}_{3}$ \\
\hline GW & 6 эก: & fun TMA TES \\
\hline & 105 & Vert IAE TEG \\
\hline & 58 & Vent $\mathrm{AsH}_{3}$ \\
\hline & $1 s$ & Ruth $\mathrm{FH}_{3}$ \\
\hline Earter & $18 \mathrm{nth}$ & Pun IM \\
\hline \multicolumn{3}{|c|}{ Sequence 9} \\
\hline Bartiog & $18 \mathrm{hm}$ & \\
\hline & $2 s$ & Vent TMV: \\
\hline & 15 & Vent $\mathrm{PH}_{3}, \mathrm{Run} \mathrm{AsH}_{3}$ \\
\hline $\mathrm{QWN}$ & $6 \mathrm{~nm}$ & RAMA THG TEG \\
\hline & 25 & Vent TWAL, TER \\
\hline Bamrier & $16 \mathrm{Am}$ & 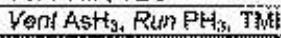 \\
\hline
\end{tabular}

\begin{tabular}{|c|c|c|}
\hline \multicolumn{3}{|c|}{ Sequence C } \\
\hline Baptier & $18 \mathrm{~nm}$ & \\
\hline & $5 s$ & Vend TBMI \\
\hline & 45 & 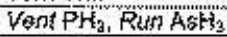 \\
\hline QW & $6 \mathrm{nth}$ & Ru/ ThUE, TEG \\
\hline & 105 & VEnt TML, TES \\
\hline & 105 & Vent AsH ${ }_{3}, R_{R T} P_{r_{3}}$ \\
\hline Bartier & $1 B$ जाI & Rum TM \\
\hline
\end{tabular}

Segurace D

\begin{tabular}{|c|c|c|}
\hline Barrier & 18 ก采 & \\
\hline & 10 s & Vent TMI \\
\hline & 58 & $\mathrm{VentP}_{3}$ \\
\hline olv" & $1 \mathrm{~s}$ & $E_{\text {RI }}$ TMt, TEG \\
\hline QWW & $6 \mathrm{~nm}$ & Ruा Astly \\
\hline & 105 & Vent TML, TEG \\
\hline & 55 & Vent AsH \\
\hline & 16 & Rum FAt: \\
\hline Barrar & $18 \mathrm{~nm}$ & Run $\mathrm{PH}_{3}$ \\
\hline
\end{tabular}


Table II. Interface composition profiles used for finite element analysis.
Figure 1.

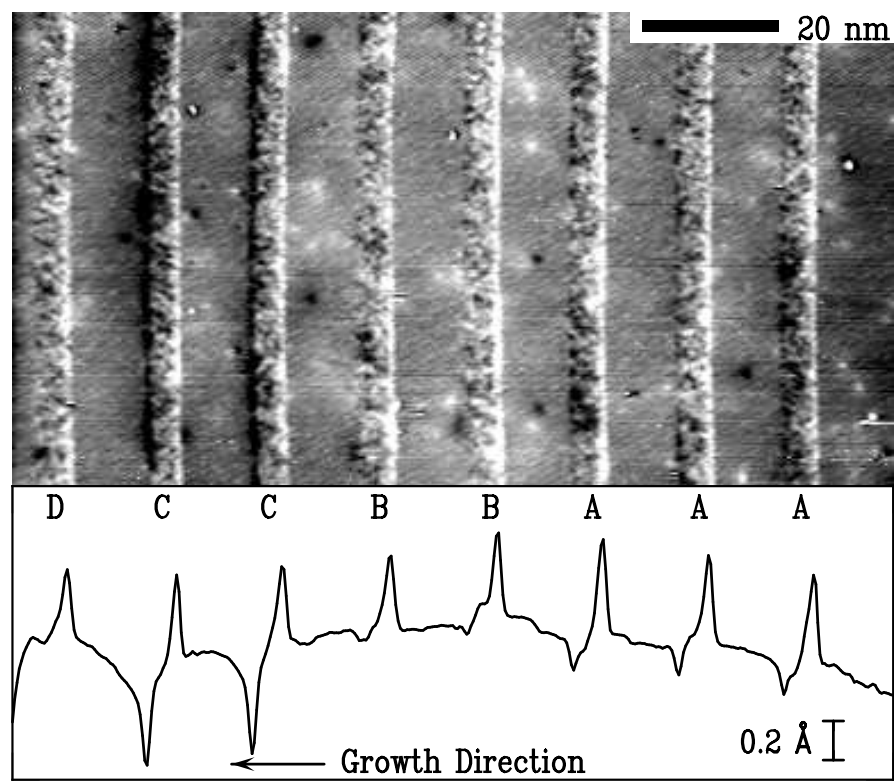


Figure 2.
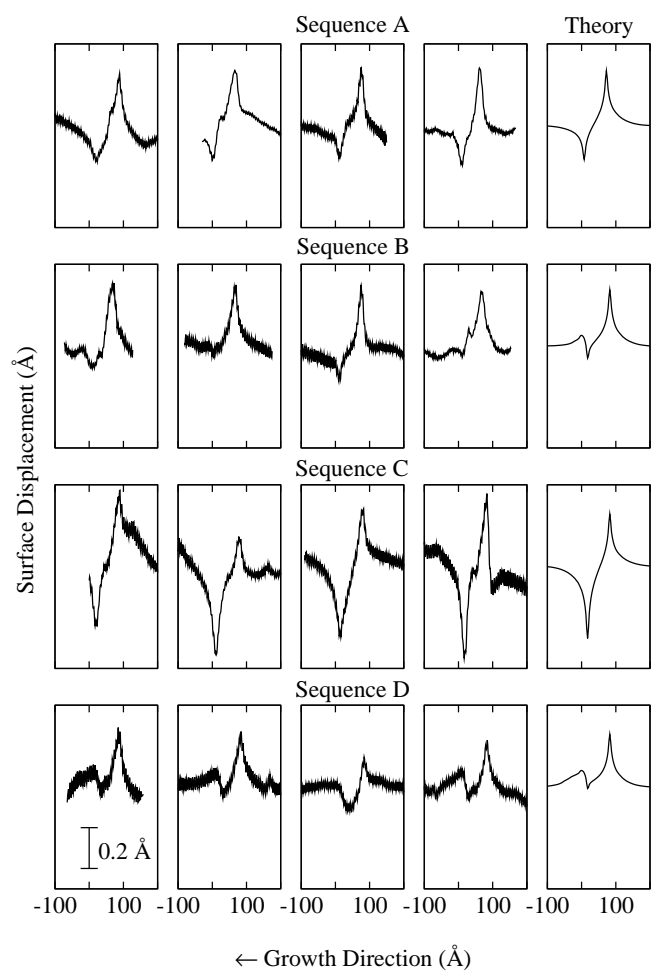

Figure 3.

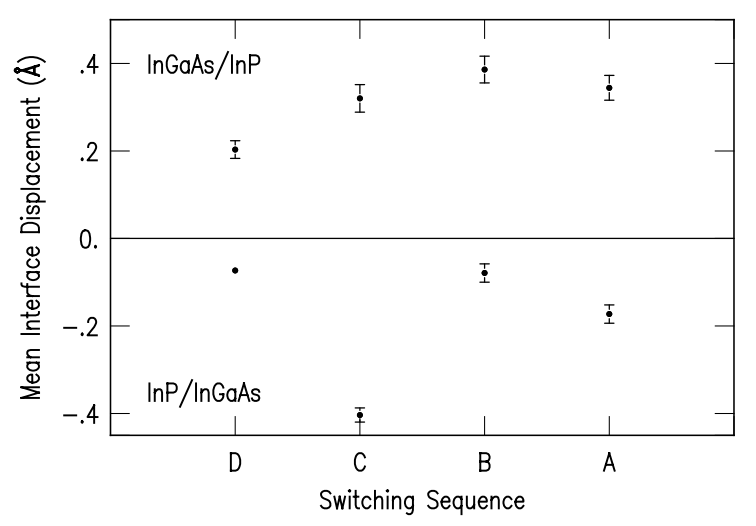


Figure 4.

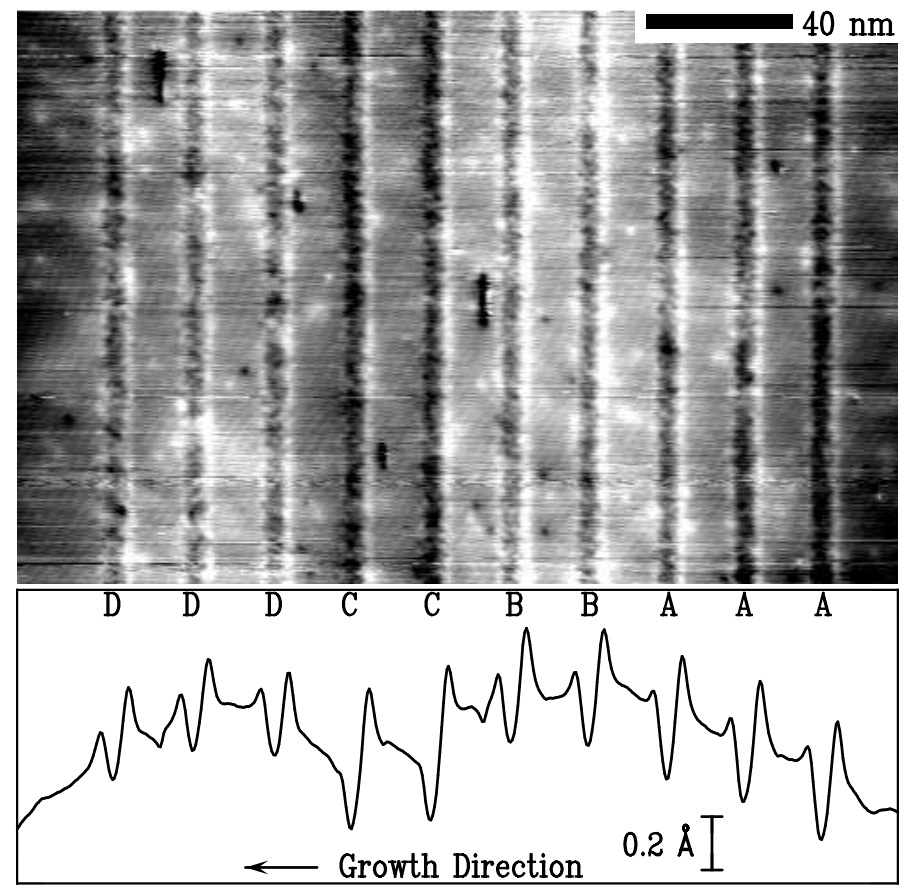

